\title{
Coloring of cozero-divisor graphs of commutative von Neumann regular rings*
}

\author{
R. NIKANDISH ${ }^{\mathrm{a}}$, M.J. NIKMEHR ${ }^{\mathrm{b}}$ AND M. BAKHTYIARI ${ }^{\mathrm{b}}$ \\ ${ }^{a}$ Department of Basic Sciences, Jundi-Shapur University of Technology, \\ P.O. BOX 64615-334, Dezful, Iran \\ r.nikandish@jsu.ac.ir \\ ${ }^{\mathrm{b}}$ Faculty of Mathematics, K.N. Toosi University of Technology, \\ P.O. BOX 16315-1618, Tehran, Iran \\ nikmehr@kntu.ac.ir m.bakhtyiari55@gmail.com
}

\begin{abstract}
Let $R$ be a commutative ring with non-zero identity. The cozero-divisor graph of $R$, denoted by $\Gamma^{\prime}(R)$, is a graph with vertices in $W^{*}(R)$, which is the set of all non-zero and non-unit elements of $R$, and two distinct vertices $a$ and $b$ in $W^{*}(R)$ are adjacent if and only if $a \notin R b$ and $b \notin R a$. In this paper, we show that the cozero-divisor graph of a von Neumann regular ring with finite clique number is not only weakly perfect but also perfect. Also, an explicit formula for the clique number is given.
\end{abstract}

\section{Introduction}

The cozero-divisor graphs associated with commutative rings, as the dual notion of zero-divisor graphs, was first introduced by Afkhami and Khashyarmanesh in [2], where they investigated some fundamental properties on the structure of this graph and the relation between cozero-divisor and zero-divisor graphs. Study of the complement of cozerodivisor graphs and characterization of commutative rings with forest, star, double-star or unicyclic cozero-divisor graphs were made bay the same authors in [3]. Planar, outerplanar and ring graph cozero-divisor graphs may be found in 44. Akbari et al. gave

${ }^{*}$ Key Words: Cozero-divisor graph; Von Neumann regular ring; Clique number; Chromatic number; Perfect graph.

2010 Mathematics Subject Classification: 05C69; 13A15; 13E05; $16 \mathrm{E} 50$. 
further results on rings with forest cozero-divisor graphs and diameter of cozero-divisor graphs associated with $R[x]$ and $R[[x]]$ (see [6]). The cozero-divisor graph has also been studied in several other papers (e.g., [5, 7, 8, 12]). In this paper, we deal with the coloring cozero-divisor graphs problem. Interested readers may find some methods in coloring of graphs associated with rings in [1, 13]. First we recall some terminology and notation.

Throughout this paper, all rings are assumed to be commutative with identity. We denote by $\operatorname{Max}(R), U(R), W(R)$ and $\operatorname{Nil}(R)$, the set of all maximal ideals of $R$, the set of all invertible elements of $R$, the set of all non-unit elements of $R$ and the set of all nilpotent elements of $R$, respectively. For a subset $T$ of a ring $R$ we let $T^{*}=T \backslash\{0\}$. The $\operatorname{ring} R$ is said to be reduced if it has no non-zero nilpotent element. The ring $R$ is called von Neumann regular if for every $r \in R$, there exists an $s \in R$ such that $r=r^{2} s$. The krull dimension of $R$, denoted by $\operatorname{dim}(R)$, is the supremum of the lengths of all chains of prime ideals. For any undefined notation or terminology in ring theory, we refer the reader to 9 .

Let $G=(V, E)$ be a graph, where $V=V(G)$ is the set of vertices and $E=E(G)$ is the set of edges. By $\bar{G}$, we mean the complement graph of $G$. We write $u-v$, to denote an edge with ends $u, v$. If $U \subseteq V(G)$, then by $N(U)$ we mean the set of all neighbors of $U$ in $G$. A graph $H=\left(V_{0}, E_{0}\right)$ is called a subgraph of $G$ if $V_{0} \subseteq V$ and $E_{0} \subseteq E$. Moreover, $H$ is called an induced subgraph by $V_{0}$, denoted by $G\left[V_{0}\right]$, if $V_{0} \subseteq V$ and $E_{0}=\left\{\{u, v\} \in E \mid u, v \in V_{0}\right\}$. Also $G$ is called a null graph if it has no edge. A clique of $G$ is a maximal complete subgraph of $G$ and the number of vertices in the largest clique of $G$, denoted by $\omega(G)$, is called the clique number of $G$. For a graph $G$, let $\chi(G)$ denote the vertex chromatic number of $G$, i.e., the minimal number of colors which can be assigned to the vertices of $G$ in such a way that every two adjacent vertices have different colors. A graph $G$ is said to be weakly perfect if $\omega(G)=\chi(G)$. A perfect graph $G$ is a graph in which every induced subgraph is weakly perfect. For any undefined notation or terminology in graph theory, we refer the reader to [14].

Let $R$ be a commutative ring with nonzero identity. The cozero-divisor graph of $R$, denoted by $\Gamma^{\prime}(R)$, is a graph with the vertex set $W^{*}(R)$ and two distinct vertices $a$ and $b$ in $W^{*}(R)$ are adjacent if and only if $a \notin R b$ and $b \notin R a$. In this paper, it is shown that the cozero-divisor graph of a von Neumann regular ring with finite clique number is weakly perfect. Moreover, an explicit formula for the clique number is given. Finally, we 
strengthen this result; Indeed it is proved that this graph is perfect.

\section{Clique and Chromatic Number of $\Gamma^{\prime}(R)$}

Let $R$ be a von Neumann regular ring and $\omega\left(\Gamma^{\prime}(R)\right)<\infty$. The main of this section is to show that $\omega\left(\Gamma^{\prime}(R)\right)=\chi\left(\Gamma^{\prime}(R)\right)=\left(\begin{array}{c}n \\ {[n / 2]}\end{array}\right)$, where $n=|\operatorname{Min}(R)|$. First, we need a series of lemma.

Lemma 2.1 Let $R$ be a ring. If $\omega\left(\Gamma^{\prime}(R)\right)<\infty$, then $R$ is a Noetherian ring.

Proof. It is enough to show that every ideal of $R$ is finitely generated. Suppose to the contrary, there exists an ideal $I$ of $R$ which is generate by the set $\left(x_{i}\right)_{i \in \Lambda}$, where $|\Lambda|=\infty$ and it is not generate by the set $\left(x_{i}\right)_{i \in \Upsilon}$, where $\Upsilon=\Lambda \backslash\{i\}$, for every $i \in \Lambda$. Thus $x_{i} \notin R x_{j}$ and $x_{j} \notin R x_{i}$, for every two distinct elements $i, j \in \Lambda$. Hence the set $\left(x_{i}\right)_{i \in \Lambda}$ is a clique of $\Gamma^{\prime}(R)$ and so $\omega\left(\Gamma^{\prime}(R)\right)=\infty$, which is a contradiction. Therefore, every ideal of $R$ is finitely generated.

Lemma 2.2 Let $R$ be a von Neumann regular ring. If $\omega\left(\Gamma^{\prime}(R)\right)<\infty$, then $R \cong$ $F_{1} \times \cdots \times F_{n}$, where every $F_{i}$ is a field and $|\operatorname{Min}(R)|=n$.

Proof. By [11, Theorem 3.1], $R$ is a reduced $\operatorname{ring}$ and $\operatorname{dim}(R)=0$. Moreover, by Lemma 2.1, $R$ is a Noetherian ring. Thus $R$ is a reduced Artinian ring. The result now follows from [9, Theorem 8.7].

Lemma 2.3 Let $R$ be a ring. Then the following statements are equivalent.

(1) $a-b$ is an edge of $\Gamma^{\prime}(R)$.

(2) $R a \nsubseteq R b$ and $R b \nsubseteq R a$.

Proof. It is straightforward.

Lemma 2.4 Let $R$ be a ring and $x, y \in V\left(\Gamma^{\prime}(R)\right)$ such that $R a=R b$. Then $N(a)=$ $N(b)$.

Proof. Suppose that $c \in N(a)$. By Lemma 2.3, $R a \nsubseteq R c$ and $R c \nsubseteq R a$. Since $R a=R b$, we deduce that $R b \nsubseteq R c$ and $R c \nsubseteq R b$ and thus by Lemma 2.3, $c \in N(b)$. Hence $N(a) \subseteq N(b)$. Similarly, $N(b) \subseteq N(a)$, as desired. 
Lemma 2.5 Let $2 \leq n<\infty$ be an integer and $R=\mathbb{Z}_{2} \times \cdots \times \mathbb{Z}_{2}$ ( $n$ times $)$. Then

$$
\omega\left(\Gamma^{\prime}(R)\right)=\chi\left(\Gamma^{\prime}(R)\right)=\left(\begin{array}{c}
n \\
{[n / 2]}
\end{array}\right) .
$$

Proof. Let $x=\left(x_{1}, \ldots, x_{n}\right) \in V\left(\Gamma^{\prime}(R)\right)$. Obviously, $x_{i}=0$ for some $i \in\{1, \ldots, n\}$. Let $N Z C(x)$ be the number zero $x_{i}$ 's in $x$, for every $x=\left(x_{1}, \ldots, x_{n}\right) \in V\left(\Gamma^{\prime}(R)\right)$. Clearly, $1 \leq N Z C(x) \leq n-1$, for every $x=\left(x_{1}, \ldots, x_{n}\right) \in V\left(\Gamma^{\prime}(R)\right)$. For every $1 \leq i \leq n-1$, let

$$
A_{i}=\left\{x=\left(x_{1}, \ldots, x_{n}\right) \in V\left(\Gamma^{\prime}(R)\right) \mid N Z C(x)=i\right\} .
$$

It is easily seen that $V\left(\Gamma^{\prime}(R)\right)=\cup_{i=1}^{n-1} A_{i}$ and $A_{i} \cap A_{j}=\varnothing$, for every $i \neq j$ and so $\left\{A_{1}, \ldots, A_{n-1}\right\}$ is a partition of $V\left(\Gamma^{\prime}(R)\right)$. We show that $\Gamma^{\prime}(R)\left[A_{i}\right]$ is a complete (induced) subgraph of $\Gamma^{\prime}(R)$, for every $1 \leq i \leq n-1$. Let $x=\left(x_{1} \ldots, x_{n}\right), y=\left(y_{1}, \ldots, y_{n}\right) \in A_{i}$, for some $1 \leq i \leq n-1$ and $x \neq y$. Since $N Z C(x)=N Z C(y)$, there exist $1 \leq i \neq j \leq n$ such that $x_{i}=0, y_{i}=1$ and $x_{j}=1, y_{j}=0$. This implies that $x \notin R y$ and $y \notin R x$ and so $x$ and $y$ are adjacent. Hence $\Gamma^{\prime}(R)\left[A_{i}\right]$ is a complete (induced) subgraph of $\Gamma^{\prime}(R)$, for every $1 \leq i \leq n-1$. Furthermore, $\left|A_{i}\right|=\left(\begin{array}{c}n \\ i\end{array}\right)$, for every $1 \leq i \leq n$ and $\left|A_{t}\right| \geq\left|A_{i}\right|$, for every $1 \leq i \leq n-1$, where $t=[n / 2]$. Let $i \neq j$ and $i<j<t$. Then $\left|A_{i}\right| \leq\left|A_{j}\right|$ and for every $x \in A_{i}$ there exists a vertex $y \in A_{j}$ such that $R y \subseteq R x$. Thus by Lemma 2.3, $x$ is not adjacent to $y$ (by replacing one of the zero components of $y \in A_{j}$ by 1 , we have $x \in A_{i}$ ). Hence

$$
\omega\left(\Gamma^{\prime}(R)\left[\cup_{i=1}^{t} A_{i}\right]\right)=\chi\left(\Gamma^{\prime}(R)\left[\cup_{i=1}^{t} A_{i}\right]\right)=\left(\begin{array}{c}
n \\
t
\end{array}\right) .
$$

Similarly,

$$
\omega\left(\Gamma^{\prime}(R)\left[\cup_{i=t}^{n-1} A_{i}\right]\right)=\chi\left(\Gamma^{\prime}(R)\left[\cup_{i=t}^{n-1} A_{i}\right]\right)=\left(\begin{array}{l}
n \\
t
\end{array}\right) .
$$

Indeed, there are enough colors in $\Gamma^{\prime}(R)\left[A_{t}\right]$ to color $\Gamma^{\prime}(R)$. Thus

$$
\omega\left(\Gamma^{\prime}(R)=\chi\left(\Gamma^{\prime}(R)\right)=\left(\begin{array}{l}
n \\
t
\end{array}\right) .\right.
$$

Remark 2.1 Let $G$ be a graph and $x \in V(G)$. If there exists a vertex $y \in V(G)$ which is not adjacent to $x$ and $N(x)=N(y)$, then $\omega(G)=\omega(G \backslash\{x\})$ and $\chi(G)=\chi(G \backslash\{x\})$.

We are now in a position to state our main result of this section. 
Theorem 2.1 Let $R$ be a von Neumann regular ring and $|\operatorname{Min}(R)|=n$. If $\left|\omega\left(\Gamma^{\prime}(R)\right)\right|<$ $\infty$, then

$$
\omega\left(\Gamma^{\prime}(R)\right)=\chi\left(\Gamma^{\prime}(R)\right)=\left(\begin{array}{c}
n \\
{[n / 2]}
\end{array}\right)
$$

Proof. By Lemma 2.2, $R \cong F_{1} \times \cdots \times F_{n}$, where $F_{i}$ is a field, for every $1 \leq i \leq n<\infty$. Let

$$
A=\left\{\left(x_{1}, \ldots, x_{n}\right) \in V\left(\Gamma^{\prime}(R)\right) \mid x_{i} \in\{0,1\} \text { for every } 1 \leq i \leq n\right\} .
$$

Consider the following claims:

Claim 1. $\omega\left(\Gamma^{\prime}(R)[A]\right)=\omega\left(\Gamma^{\prime}(R)\right)$ and $\chi\left(\Gamma^{\prime}(R)[A]\right)=\chi\left(\Gamma^{\prime}(R)\right)$.

Suppose that $x=\left(x_{1}, \ldots, x_{n}\right)$ and $y=\left(y_{1}, \ldots, y_{n}\right)$ are vertices of $\Gamma^{\prime}(R)$. Define the relation $\sim$ on $V\left(\Gamma^{\prime}(R)\right)$ as follows: $x \sim y$, whenever " $x_{i}=0$ if and only if $y_{i}=$ 0 ", for every $1 \leq i \leq n$. Obviously, $\sim$ is an equivalence relation on $V\left(\Gamma^{\prime}(R)\right)$. Thus $V\left(\Gamma^{\prime}(R)\right)=\cup_{i=1}^{2^{n}-2}[x]_{i}$, where $[x]_{i}$ is the equivalence class of $x_{i}$ (We note that the number of equivalence classes is $\left.2^{n}-2\right)$. Let $[x]$ be a equivalence class of $x$. Then $|[x] \cap A|=1$ and so one may choose $a \in[x] \cap A$ and $b \in[x] \backslash\{a\}$. Since $R a=R b$, by Lemma 2.4. $N(a)=N(b)$. By Remark 3.1, $\omega\left(\Gamma^{\prime}(R)\right)=\omega\left(\Gamma^{\prime}(R) \backslash\{b\}\right)$ and $\chi\left(\Gamma^{\prime}(R)\right)=\chi\left(\Gamma^{\prime}(R) \backslash\{b\}\right)$. If we continue this procedure for $\left|V\left(\Gamma^{\prime}(R)\right) \backslash A\right|$ times, then we get $\omega\left(\Gamma^{\prime}(R)[A]\right)=\omega\left(\Gamma^{\prime}(R)\right)$ and $\chi\left(\Gamma^{\prime}(R)[A]\right)=\chi\left(\Gamma^{\prime}(R)\right)$.

Claim 2. $\omega\left(\Gamma^{\prime}(R)[A]\right)=\omega\left(\Gamma^{\prime}(S)\right)$ and $\chi\left(\Gamma^{\prime}(R)[A]\right)=\chi\left(\Gamma^{\prime}(S)\right)$, where $S=\mathbb{Z}_{2} \times \cdots \times \mathbb{Z}_{2}$ ( $n$ times).

Let $x=\left(x_{1}, \ldots, x_{n}\right) \in S \backslash\{0,1\}$ and $y=\left(y_{1}, \ldots, y_{n}\right) \in A$. Consider the map $\varphi$ : $S \backslash\{0,1\} \longrightarrow A$ defined by the rule $\varphi(x)=y$, whenever $x_{i}=0$ if and only if $y_{i}=0$. It is not hard to check that $\varphi$ is well-defined, bijective and if $x, y \in S \backslash\{0,1\}$ such that $x$ is adjacent $y$, then $\varphi(x)$ is adjacent $\varphi(y)$. This implies that $\Gamma^{\prime}(S) \cong \Gamma^{\prime}(R)[A]$ and thus $\omega\left(\Gamma^{\prime}(R)[A]\right)=\omega\left(\Gamma^{\prime}(S)\right)$ and $\chi\left(\Gamma^{\prime}(R)[A]\right)=\chi\left(\Gamma^{\prime}(S)\right)$.

By Claims 1,2 and Lemma 2.5.

$$
\omega\left(\Gamma^{\prime}(R)\right)=\chi\left(\Gamma^{\prime}(R)\right)=\omega\left(\Gamma^{\prime}(R)[A]\right)=\chi\left(\Gamma^{\prime}(R)[A]\right)=\omega\left(\Gamma^{\prime}(S)\right)=\chi\left(\Gamma^{\prime}(S)\right)=\left(\begin{array}{c}
n \\
{[n / 2]}
\end{array}\right) .
$$

We close this section with the following proposition.

Proposition 2.1 Let $R$ be a ring which is not an integral domain. If $\left|\omega\left(\Gamma^{\prime}(R)\right)\right|<\infty$, then $\Gamma^{\prime}(R)$ is a null graph if and only if $(R, \mathfrak{m})$ is local ring and $\mathfrak{m}$ is principal. 
Proof. First, suppose that $\Gamma^{\prime}(R)$ is a null graph. If $R$ is not local, then one may choose $x \in \mathfrak{m}_{1} \backslash \mathfrak{m}_{2}$ and $y \in \mathfrak{m}_{2} \backslash \mathfrak{m}_{1}$, where $\mathfrak{m}_{1}, \mathfrak{m}_{2} \in \operatorname{Max}(R)$. Since $x$ is not adjacent to $y$, we find a contradiction. Thus $(R, \mathfrak{m})$ is local ring. Also, by a similar argument to the proof of Lemma 2.1, one may show that $\mathfrak{m}$ is principal.

To prove the converse, suppose that $(R, \mathfrak{m})$ is a local ring and $\mathfrak{m}$ is principal. We show that $\operatorname{dim}(R)=0$. It is enough to show that $\mathfrak{m} \in \operatorname{Min}(R)$. Assume that $\mathfrak{p} \subseteq \mathfrak{m}$, for some $\mathfrak{p} \in \operatorname{Min}(R)$. Since $R$ is not an integral domain, $\mathfrak{p} \neq(0)$ and so we may pick $0 \neq a \in \mathfrak{p}$. Since $\mathfrak{m}$ is principal, $\mathfrak{m}=R x$, for some $x \in R$. If $x \in \mathfrak{p}$, then $\mathfrak{p}=\mathfrak{m}$ and thus $\operatorname{dim}(R)=0$. So let $x \notin \mathfrak{p}$. Since $\mathfrak{p} \subseteq \mathfrak{m}, a=r_{1} x$ for some $r_{1} \in R$. Also $x \notin \mathfrak{p}$ implies that $r_{1} \in \mathfrak{p}$ and thus $r_{1}=r_{2} x$, for some $r_{2} \in R$. Hence $a=r_{2} x^{2}$ and so $a \in \mathfrak{m}^{2}$. If we continue this procedure, then $a \in \mathfrak{m}^{n}$, for every positive integer $n$. Therefore $a \in \cap_{n=1}^{\infty} \mathfrak{m}^{n}$. This, together with [9, Corolary 10.19] imply that $a=0$, a contradiction. Hence $\mathfrak{p}=\mathfrak{m}$ and so $\operatorname{dim}(R)=0$. Since $R$ is Noetherian with $\operatorname{dim}(R)=0, R$ is an Artinian local ring. Finally, by [9, Proposition 8.8], every ideal of $R$ is principal and hence $\Gamma^{\prime}(R)$ is a null graph.

\section{Perfectness of $\Gamma^{\prime}(R)$}

Let $R$ be a von Neumann regular ring and $\omega\left(\Gamma^{\prime}(R)\right)<\infty$. In this section, we show that $\Gamma^{\prime}(R)$ is a perfect graph. We begin with the following celebrate result.

Lemma 3.1 ([10] The Strong Perfect Graph Theorem) A graph $G$ is perfect if and only if neither $G$ nor $\bar{G}$ contains an induced odd cycle of length at least 5.

Theorem 3.1 Let $R=\mathbb{Z}_{2} \times \cdots \times \mathbb{Z}_{2}$ ( $n$ times). Then $\Gamma^{\prime}(R)$ is perfect.

Proof. By Lemma 3.1, it is enough to prove the following claims.

Claim 1. $\Gamma^{\prime}(R)$ contains no induced odd cycle of length at least 5. Assume to the contrary,

$$
a_{1}-a_{2}-\cdots-a_{n}-a_{1}
$$

is an induced odd cycle of length at least 5 in $\Gamma^{\prime}(R)$.

By Lemma 2.3, either $R a_{1} \subseteq R a_{3}$ or $R a_{3} \subseteq R a_{1}$. We show that these two cases lead to contradictions. First assume that the case $R a_{1} \subseteq R a_{3}$ happens. We continue the proof by proving the following subclaims.

Subclaim 1. $R a_{1} \subseteq R a_{i}$, for every $3 \leq i \leq n-1$.

Clearly, $R a_{1} \subseteq R a_{3}$. By Lemma [2.3, $R a_{1} \subseteq R a_{4}$ or $R a_{4} \subseteq R a_{1}$. If $R a_{4} \subseteq R a_{1}$, then $R a_{4} \subseteq R a_{3}$, a contradiction, by Lemma 2.3. So $R a_{1} \subseteq R a_{4}$. Again, by Lemma 
2.3. $R a_{1} \subseteq R a_{5}$ or $R a_{5} \subseteq R a_{1}$. If $R a_{5} \subseteq R a_{1}$, then since $R a_{1} \subseteq R a_{4}, R a_{5} \subseteq R a_{4}$, a contradiction. Thus $R a_{1} \subseteq R a_{5}$. Similarly, $R a_{1} \subseteq R a_{i}$, for every $6 \leq i \leq n-1$.

Subclaim 2. $R a_{2} \subseteq R a_{i}$, for every $4 \leq i \leq n$. Obviously, $R a_{1} \subseteq R a_{4}$, by the Subclaim 1. By Lemma 2.3, $R a_{2} \subseteq R a_{4}$ or $R a_{4} \subseteq R a_{2}$. If $R a_{4} \subseteq R a_{2}$, then $R a_{1} \subseteq R a_{2}$, a contradiction. So $R a_{2} \subseteq R a_{4}$. Next, we show that $R a_{2} \subseteq R a_{5}$. If $R a_{5} \subseteq R a_{2}$, then since $R a_{2} \subseteq R a_{4}$, we deduce that $R a_{5} \subseteq R a_{4}$, a contradiction. Therefore $R a_{2} \subseteq R a_{5}$. Similarly, $R a_{2} \subseteq R a_{i}$, for every $6 \leq i \leq n$.

Now, using Subclaims 1 and 2, we show that $R a_{3} \subseteq R a_{1}$. By Lemma 2.3, $R a_{3} \subseteq R a_{5}$ or $R a_{5} \subseteq R a_{3}$. If $R a_{5} \subseteq R a_{3}$, then by Subclaim 2, $R a_{2} \subseteq R a_{3}$, a contradiction. Thus $R a_{3} \subseteq R a_{5}$. We show that $R a_{3} \subseteq R a_{6}$. If $R a_{6} \subseteq R a_{3}$, then by Subcase $2, R a_{2} \subseteq R a_{3}$, a contradiction. So $R a_{3} \subseteq R a_{6}$. Similarly, $R a_{3} \subseteq R a_{i}$, for every $7 \leq i \leq n$. Since $R a_{1} \subseteq R a_{3}, R a_{1} \subseteq R a_{i}$, for every $5 \leq i \leq n$, i.e., $R a_{1} \subseteq R a_{n}$, a contradiction. Thus $R a_{3} \subseteq R a_{1}$ and this contradicts Subclaim 1. Therefore, $\Gamma^{\prime}(R)$ contains no induced odd cycle of length at least 5 .

Claim 2. $\overline{\Gamma^{\prime}(R)}$ contains no induced odd cycle of length at least 5. Assume to the contrary,

$$
a_{1}-a_{2}-\cdots-a_{n}-a_{1}
$$

is an induced odd cycle of length at least 5 in $\overline{\Gamma^{\prime}(R)}$. By Lemma 2.3 , we may assume that $R a_{1} \subseteq R a_{2}$. If $R a_{2} \subseteq R a_{3}$, then $R a_{1} \subseteq R a_{3}$, a contradiction. Thus

$$
\begin{aligned}
& R a_{1} \subseteq R a_{2}, \\
& R a_{3} \subseteq R a_{2} .
\end{aligned}
$$

If $R a_{4} \subseteq R a_{3}$, then $R a_{4} \subseteq R a_{2}$, a contradiction. Hence $R a_{3} \subseteq R a_{4}$. If $R a_{4} \subseteq R a_{5}$, then $R a_{3} \subseteq R a_{4}$ implies that $R a_{3} \subseteq R a_{5}$, a contradiction. Thus

$$
\begin{aligned}
& R a_{3} \subseteq R a_{4}, \\
& R a_{5} \subseteq R a_{4} .
\end{aligned}
$$

Since $n$ is odd, by continuing this procedure, we find

$$
\begin{gathered}
R a_{n-2} \subseteq R a_{n-1}, \\
R a_{n} \subseteq R a_{n+1}=R a_{1} .
\end{gathered}
$$

This implies that $R a_{n} \subseteq R a_{1}$ and since $R a_{1} \subseteq R a_{2}, R a_{n} \subseteq R a_{2}$, a contradiction. Therefore, $\overline{\Gamma^{\prime}(R)}$ contains no induced odd cycle of length at least 5 . 
The proof now is complete.

Remark 3.1 Let $G$ be a graph and $x \in V(G)$. If there exists a vertex $y \in V(G)$ which is not adjacent to $x$ and $N(x)=N(y)$, then $G$ is perfect if and only if $G \backslash\{x\}$ is perfect.

We close this paper with the following result.

Theorem 3.2 Let $R$ be a von Neumann regular ring and $\omega\left(\Gamma^{\prime}(R)\right)<\infty$. Then $\Gamma^{\prime}(R)$ is a perfect graph.

Proof. Since $\left|\omega\left(\Gamma^{\prime}(R)\right)\right|<\infty$, it follows from Lemma 2.2 that $R \cong F_{1} \times \cdots \times F_{n}$, where $F_{i}$ is a field, for every $1 \leq i \leq n<\infty$. Let

$$
A=\left\{\left(x_{1}, \ldots, x_{n}\right) \in V\left(\Gamma^{\prime}(R)\right) \mid x_{i} \in\{0,1\} \text { for every } 1 \leq i \leq n\right\} .
$$

By Lemma 2.4 and Remark 3.1, it is not hard to check that $\Gamma^{\prime}(R)$ is perfect graph if and only if $\Gamma^{\prime}(R)[A]$ is perfect. In fact if

$$
a_{1}-a_{2}-\cdots-a_{n}-a_{1}
$$

is an induced odd cycle of length at least 5 in $\overline{\Gamma^{\prime}(R)}$ or $\Gamma^{\prime}(R)$, then $R a_{i} \neq R a_{j}$, for every $1 \leq i, j \leq n, i \neq j$. By the proof of Theorem 2.1 , we find that $\Gamma^{\prime}(R)[A] \cong \Gamma^{\prime}(S)$, where $S=\mathbb{Z}_{2} \times \cdots \times \mathbb{Z}_{2}$ ( $n$ times). Thus $\Gamma^{\prime}(R)$ is perfect if and only if $\Gamma^{\prime}(S)$ is perfect. The result now follows from Lemma 3.1 .

\section{References}

[1] G. Aalipour, S. Akbari, R. Nikandish, M.J. Nikmehr and F. Shaveisi. On the coloring of the annihilating-ideal graph of a commutative ring, Discrete. Math. 312 (2012) 2620-2626.

[2] M. Afkhami, K. Khashyarmanesh, The cozero-divisor graph of a commutative ring, Southeast Asian Bull. Math. 35 (2011) 753-762.

[3] M. Afkhami, K. Khashyarmanesh, On the cozero-divisor graphs of commutative rings and their complements, Bull. Malays. Math. Sci. Soc. 35 (2012) 935-944.

[4] M. Afkhami, M. Farrokhi D. G., K. Khashyarmanesh, Planar, outerplanar and ring graph cozero-divisor graphs. Ars Comb. 131 (2017) 397-406.

[5] M. Afkhami, K. Khashyarmanesh, On the cozero-divisor graphs of and comaximal graphs of commutative rings, J. Algebra Appl. 12 (2013) 1250173 [9 pages]. 
[6] S. Akbari, F. Alizadeh, S. Khojasteh, Some results on Cozero-divisor graph of a commutative ring, J. Algebra Appl. 13 (2014) 1350113 [14 pages].

[7] S. Akbari, S. Khojasteh, Commutative rings whose cozero-divisor graphs unicyclic or of bounded degree, Comm. Algebra 42 (2013) 1594-1605.

[8] H. Ansari-Toroght, F. Farshadifar, Sh.Habibi, The cozero-divisor graph relative to finitely generated modules, Miskolc Mathematical Notes 14 (2013) 749-756.

[9] M. F. Atiyah, I.G. Macdonald, Introduction to Commutative Algebra, Addison-Wesley Publishing Company, 1969.

[10] R. Diestel, Graph Theory, NY, USA: Springer-Verlag, 2000.

[11] J. A. Huckaba, Commutative Rings With Zero Divisors, 2nd ed., Prentice Hall, Upper Saddle River (1988).

[12] S. Kavitha, R. Kala, On the genus of graphs from commutative rings, AKCE International Journal of Graphs and Combinatorics, 14 (2017) 27-34.

[13] H. R. Maimani, M. R. Pournaki, S. Yassemi, A class of weakly perfect graphs, Czech. Math. Journal 60 (2010) 1037-1041.

[14] D. B. West, Introduction to Graph Theory, 2nd ed., Prentice Hall, Upper Saddle River (2001). 